\title{
Saccharomyces cerevisiae ER membrane protein complex subunit 4 (EMC4) plays a crucial role in elF2B-mediated translation regulation and survival under stress conditions
}

Sonum Sharma', Anuradha Sourirajan', David J. Baumler ${ }^{2,3,4}$ and Kamal Dev*

\begin{abstract}
Background: Eukaryotic initiation factor 2B (elF2B) initiates and regulates translation initiation in eukaryotes. elF2B gene mutations cause leukoencephalopathy called vanishing white matter disease (VWM) in humans and slow growth $\left(\mathrm{Slg}^{-}\right)$and general control derepression $\left(\mathrm{Gcd}^{-}\right)$phenotypes in Saccharomyces cerevisiae.

Results: To suppress elF2B mutations, S. cerevisiae genomic DNA library was constructed in high-copy vector (YEp24) and transformed into elF2B mutant S. cerevisiae strains. The library was screened for wild-type genes rescuing S. cerevisiae (Slg-) and $\left(\mathrm{Gcd}^{-}\right)$phenotypes. A genomic clone, Suppressor-I (Sup-I), rescued S. cerevisiae Slg and $\mathrm{Gcd}^{-}$phenotypes (gcd7-201 gcn24). The YEp24/Sup-I construct contained truncated TAN1, full length EMC4, full length YGL230C, and truncated SAP4 genes. Full length EMC4 (chaperone protein) gene was sub-cloned into pEG (KG) yeast expression vector and overexpressed in gcd7-201 gcn2 $\triangle$ strain which suppressed the $\mathrm{Slg}^{-}$and Gcd ${ }^{-}$ phenotype. A GST-Emc4 fusion protein of $47 \mathrm{kDa}$ was detected by western blotting using a-GST antibodies. Suppression was specific to gcd7-201 gcn2 $\Delta$ mutation in elF2Bß and Gcd1-502 gcn2 $\Delta$ in elF2By subunit. Emc4p overexpression also protected the wild type and mutant (gcd7-201 gcn2 4 , GCD7 gcn2 2 , and GCD7 GCN2 $\Delta$ ) strains from $\mathrm{H}_{2} \mathrm{O}_{2}$, ethanol, and caffeine stress.
\end{abstract}

Conclusions: Our results suggest that Emc4p is involved in elF2B-mediated translational regulation under stress and could provide an amenable tool to understand the elF2B-mediated defects.

Keywords: S. cerevisiae, elF2B, WWM, Suppression, Identification, Emc4p, General Control Derepressed (GCD), General Control Nonderepressible (GCN)

\section{Background}

Eukaryotic initiation factor 2B (eIF2B) a heterodecameric complex of five non-identical protein subunits $(\alpha-\varepsilon)$ initiates/regulates translation [1]. $\alpha, \beta$, and $\delta$ subunits of eF2B constitute regulatory sub complex, while the $\gamma$ and $\varepsilon$ subunits form catalytic sub complex [2]. eIF2B initiates

\footnotetext{
* Correspondence: kamaldevbhardwaj1969@gmail.com

${ }^{1}$ Faculty of Applied Sciences and Biotechnology, Shoolini University, Solan, Himachal Pradesh 173212, India

Full list of author information is available at the end of the article
}

translation by catalyzing the GDP-GTP exchange on its substrate, eukaryotic initiation factor 2 (eIF2). Under stress, eIF2B tightly binds to the phosphorylated eIF2 [2-4] which reduces eIF2B activity, and a transcriptionactivating factor GCN4 in S. cerevisiae and ATF4 in humans are translated $[5,6]$ inducing various stress response genes [7]. Mutations in eIF2B subunits cause a neurodegenerative disease, called VWM (leukoencephalopathy with vanishing white matter) [8-10]. In VWM patients, eIF2B GEF activities are generally lower than

\section{Springer Open}

() The Author(s). 2020 Open Access This article is licensed under a Creative Commons Attribution 4.0 International License, which permits use, sharing, adaptation, distribution and reproduction in any medium or format, as long as you give appropriate credit to the original author(s) and the source, provide a link to the Creative Commons licence, and indicate if changes were made. The images or other third party material in this article are included in the article's Creative Commons licence, unless indicated otherwise in a credit line to the material. If material is not included in the article's Creative Commons licence and your intended use is not permitted by statutory regulation or exceeds the permitted use, you will need to obtain permission directly from the copyright holder. To view a copy of this licence, visit http://creativecommons.org/licenses/by/4.0/. 
normal [11] and is insensitive to eIF2 (loss of eIF2B-eIF2 interaction) [12-14]. Low eIF2B activity induces GCN4/ ATF4 even in absence of eIF2 phosphorylation [15-19] and induces stress-like conditions. Neurological disorder further provokes additional stress and white matter deterioration.

Regulatory subunits of eIF2B are important for eI2BeIF2 interaction under normal and stress conditions. Archeal eIF2B interacts with eukaryotic eIF2 $\alpha$ and eIF2B $\alpha$ indicating the importance of regulatory subunits [20]. eIF2B $\beta$ subunit binds eIF2 which is important for eIF2-eIF2B interaction and translation regulation [21]. During integrated stress response, mutations in eIF2B $\beta$ subunit suppress translation and cause delay in the recovery [22]. Identifying extragenic suppressors, modulators (proteins/chemicals) of mutated eIF2B regulatory subunits, may be useful in curing VWM disease. The chemical modulators, activating either GCN4 or suppressing eIF2B mutations, have been previously identified [23]. The goal of our study was to identify the $S$. cerevisiae protein that interacts with mutated eIF2B subunit and suppresses the mutation.

eIF2B mutant S. cerevisiae strains with deletion of protein kinase Gcn2p (phosphorylates eIF2 $\alpha$ ) gene give general control derepression phenotype ( $\mathrm{Gcd}^{-}$phenotype) and slow growth $\left(\mathrm{Slg}^{-}\right)$phenotype. In $\mathrm{Gcd}^{-}$phenotype, GCN4 is activated even in absence of eIF2 $\alpha$ phosphorylation. The qualitative measurement of eIF2B activity and GCN4 activation in $g c n 2 \Delta$ strains can be measured in vivo on 3-amino triazole (3-AT) plates. 3-Amino triazole (3-AT) is a histidine analog and causes amino acid (histidine) starvation in $S$. cerevisiae-activating Gcn2p kinase and Gcn $4 p$ expression. If there are mutations in eIF2B subunit genes in gcn $2 \Delta$ strains, the GEF activity of eIF2B is reduced. This reduction in eIF2B GEF activity helps in the growth of $S$. cerevisiae strains on medium containing 3-AT. This assay is used for indirect expression of Gcn4p.

In the present study, overexpression of a wild-type $S$. cerevisiae chaperone protein ER transmembrane complex 4 (Emc4p) rescued both the $\mathrm{Slg}^{-}$and $\mathrm{Gcd}^{-}$ phenotypes of $S$. cerevisiae strains containing mutations either in $\beta$ ( $g c d 7-201)$ or $\gamma(g c d 1-502)$ subunits. Here we observed that Emc4p overexpression confers resistant to the $\mathrm{H}_{2} \mathrm{O}_{2}$, ethanol, and caffeine stress, in mutant or wild-type cells. We proposed a model that Emc4p by its chaperone activity folds and stabilize the destabilized and unfolded eIF2B $\beta$ and eIF2B $\gamma$ subunits. However, it is unclear why Emc4p cannot suppress the mutations in other subunits of eIF2B. But this clearly suggests that interaction of both the subunits eIF2B $\beta$ and eIF2 $\gamma$ with each other is critical for eIF2B activity, and mutations in any of these subunits can cause VWM disease.

\section{Methods}

All the chemicals and reagents were of molecular biology grade procured from Thermo scientific, Himedia Labs, India; MP Biomedicals, USA; Fermentas Inc. USA; and Bio-Rad. USA.

\section{S. cerevisiae strains and plasmids}

S. cerevisiae strains employed in this study (Table S1) were cultured on YPD agar or liquid medium. S. cerevisiae transformants were selected on synthetic complete (SC) medium lacking uracil and supplemented with glucose/galactose/raffinose. S. cerevisiae strains were incubated at $30^{\circ} \mathrm{C}$. E. coli strain $\mathrm{DH} 5 \alpha$ was used for $S$. cerevisiae genomic DNA library construction and plasmid isolation.

YEp24 (high copy shuttle vector) and pEG(KG) (yeast expression vector) were used for cloning and expression of $S$. cerevisiae genes respectively. Nutrient broth (NB, Himedia Labs, Mumbai) with $100 \mu \mathrm{g} / \mathrm{ml}$ ampicillin was used to culture the E. coli strain DH5 $\alpha$ harboring YEp24 or pEG(KG) at $37^{\circ} \mathrm{C}$. Plasmid DNA of YEp24 and pEG(KG) were isolated and used in transformations of yeast strains $[24,25]$.

\section{Construction of S. cerevisiae genomic DNA library and transformation into elF2B mutant $S$. cerevisiae strains}

Genomic DNA from S. cerevisiae strain H4 (Table S1) was isolated and partially digested with Sau3AI enzyme [24]. Fifty nanograms of partially digested and gel purified (gel purification kit Thermo-scientific) genomic DNA was ligated with $20 \mu \mathrm{g}$ of YEp24 vector at BamHI site using T4 DNA ligase [26]. After ligation at $16^{\circ} \mathrm{C}$ for $16 \mathrm{~h}, E$. coli strain $\mathrm{DH} 5 \alpha$ was transformed with the ligation mix by heat shock method [24]. The transformation mix was plated on NA medium containing ampicillin $(100 \mu \mathrm{g} / \mathrm{ml})$. Transformations were selected against ampicillin resistance on NA medium containing ampicillin and were pooled into three groups named as pool-I, pool-II, and pool-III.

Plasmid DNA isolation from three pools indicating 13,575 cfu (colony-forming units) of transformants of DH5 $\alpha$ was done [24]. Plasmids isolated from all three pools or vector (YEp24) alone were transformed into $S$. cerevisiae eIF2B mutant strains (Figure S1). The wildtype strains were transformed with YEp24 vector alone using LiAc method [25]. The nomenclature used for various $S$. cerevisiae strains used in this study is given in (Table S2). Transformation mix was plated on synthetic complete (SC) medium containing $2 \%$ glucose lacking uracil. SC mixture lacking uracil was used as a dropout supplement to select transformants containing uracilbased plasmid. eIF2B mutant $S$. cerevisiae transformants with normal colony size were compared to that of vector-transformed eIF2B mutant strains and wild-type 
strains by streaking and spot assay on synthetic complete (SC) medium containing $2 \%$ glucose lacking uracil [27].

\section{Screening of suppressor protein} eIF2B $\beta$ ( $g c d 7-201$ gcn2 $\Delta$ ) transformants showing $\mathrm{Slg}^{+}$ phenotype as that of isogenic wild type were selected and analyzed for $\mathrm{Gcd}^{+}$phenotype by spot assay on SCmedium supplemented with $30 \mathrm{mM} 3$-AT (3-amino triazole). Transformants showing $\mathrm{Slg}^{+}$and $\mathrm{Gcd}^{+}$phenotype were further screened by spot assay of 10 -fold serially diluted culture and by streaking.

Plasmid DNA from the potential $\operatorname{gcd} 7-201 \operatorname{gcn} 2 \Delta$ transformants $\left(\mathrm{Slg}^{+}, \mathrm{Gcd}^{+}\right)$were isolated [28], and $g c d 7$ $201 \operatorname{gcn} 2 \Delta$ mutant $S$. cerevisiae strain were transformed with the rescued plasmid. Simultaneously, the rescued plasmid was sequenced on both the strands at Eurofins Bangalore, (http://www.eurofins.in/) by using YEp24 vector specific primers (S7).

\section{Functional characterization of suppressor protein}

$E M C 4$ gene from rescued plasmid was amplified using gene-specific primers (Table S3) followed by sub-cloning into $\mathrm{pEG}(\mathrm{KG})$ yeast expression vector (containing a GAL1 promoter and a protease cleavable $\mathrm{N}$-terminal GST tag) at XbaI/Sall restriction sites. Gal promoter is repressed by raffinose and induced by galactose.

DH5 $\alpha$ was transformed with recombinant plasmids (100 ng) by heat shock method [24]. Rescued plasmid DNA from transformants was sequenced at Eurofins Bangalore, (http://www.eurofins.in/). An error free nucleotide sequence of EMC4 DNA was obtained. $\mathrm{pEG}(\mathrm{KG}) / E M C 4$ plasmids were transformed into $g c d 7$ $201 \operatorname{gcn} 2 \Delta$ strain by LiAc method in order to confirm the $\mathrm{Slg}^{+}$and $\mathrm{Gcd}^{+}$phenotype. The transformation mix was plated on SC medium supplemented with uracil and $2 \%$ galactose. $\operatorname{gcd} 7-201 \operatorname{gcn} 2 \Delta$ and GCD7 gcn $2 \Delta$ transformed with pEG(KG) vector alone were used as control.

Plasmid DNA isolation from the recombinant clones was done as described [28] and was transformed again in $g c d 7-201 \mathrm{gcn} 2 \Delta$. Spot assay of pEG(KG)/EMC4 transformants was performed in order to confirm the $\mathrm{Slg}^{+}$ and $\mathrm{Gcd}^{+}$phenotype. GST-EMC4-based suppression was also confirmed by eviction of pEG(KG) a uracil-based plasmid containing GST-EMC4 on 5-fluoroorotic acid (FOA) containing medium. 5-Flouroorotic acid (5-FOA) is converted to a toxic product (5-floorouracil) by URA3 gene product. Thus, $S$. cerevisiae cells containing URA3 marker cannot grow on medium containing 5-FOA but are able to grow on medium lacking uracil. Thus, FOA is used to select for the loss of vectors carrying the wildtype URA marker [29]. Colonies from FOA plate were picked and streaked on SC medium without uracil and supplemented with $2 \%$ galactose. Plates were incubated at $30^{\circ} \mathrm{C}$ for 2 days and were observed for growth phenotype.

\section{Western blot analysis}

The whole cell extract of $g c d 7-201 g c n 2 \Delta$ harboring either $\mathrm{pEG}(\mathrm{KG})$ or $\mathrm{pEG}(\mathrm{KG}) / E M C 4$ was prepared by glass bead lysis method using Fast Prep (MP Biomedicals). $\operatorname{gcd} 7-201 \operatorname{gcn} 2 \Delta$ harboring either pEG(KG) or pEG(KG)/ $E M C 4$ were incubated in $\mathrm{SC}$-medium $(5 \mathrm{ml})$ supplemented with $2 \%$ raffinose $(\mathrm{w} / \mathrm{v})$ at $30^{\circ} \mathrm{C}$ for $18 \mathrm{~h}$. Ten milliliters of SC medium supplemented with $2 \%$ raffinose $(w / v)$ was inoculated with $1 \%$ of overnight grown primary culture followed by incubation at $30^{\circ} \mathrm{C}$. At an absorbance of $\mathrm{A}_{600}$ of $\sim 0.5$, an aliquot was collected as the uninduced control, and the remaining culture was induced by $2 \%$ galactose $(w / v)$. Both induced and uninduced cultures were incubated for an additional $3 \mathrm{~h}$ at $30{ }^{\circ} \mathrm{C}$.

After incubation, cells were harvested by centrifugation $(6000 \mathrm{rpm}$ for $10 \mathrm{~min})$. Protein extraction of both induced and uninduced culture was carried using $20 \%$ tri-chloroacetic acid (TCA), and $20 \mu \mathrm{g}$ of extracted proteins were resolved on SDS-PAGE followed by the transfer to the nitrocellulose membrane (Millipore, Immobilon P $0.45 \mu \mathrm{m}$ ) by electroblotting. The blot was incubated at $4{ }^{\circ} \mathrm{C}$ for $1 \mathrm{~h}$ in blocking solution containing $5 \%$ non-fat dried milk. After incubation, membrane was further incubated with anti-GST antibodies (1:5000, Abcam) overnight at $4{ }^{\circ} \mathrm{C}$. Immunoreactive proteins were detected by using anti-rabbit IgG conjugated to horseradish peroxidase $(1: 10,000$, Abcam) for $1 \mathrm{~h}$. Blots were washed by using PBST (phosphate buffer saline containing TritonX-100) buffer. Finally, the blots were developed using enhanced chemiluminescence kit (ECL, Bio-Rad, Inc. USA).

\section{Expression of Emc4p in elF2By, elF2B $\delta$, elF2 $\varepsilon$, and GCN2 mutant $S$. cerevisiae strains}

Suppression analysis by Emc4p in other eIF2B mutants was done. eIF2B $\gamma$ (H70), eIF2B $\delta$ (H750), eIF2Be (H1792), and GCN2 (H591) mutants (Table S1) were

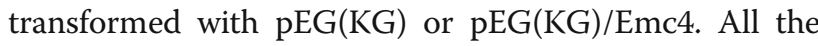
transformants were plated on SC-medium lacking uracil supplemented with $2 \%$ galactose, and the plates were incubated for 2 days at $30{ }^{\circ} \mathrm{C}$. The transformants were selected and analyzed for $\mathrm{Slg}^{+}$and $\mathrm{Gcd}^{+}$phenotype by streaking and spot assay on SC-medium lacking uracil and containing $2 \%$ galactose or SC-medium lacking uracil and containing $2 \%$ galactose and $30 \mathrm{mM} 3-\mathrm{AT}$ respectively. Plates were incubated for 2 days at $30^{\circ} \mathrm{C}$. eIF2By (H70) a $\mathrm{Ts}^{-}$mutant was also checked for suppression of temperature sensitive $\left(\mathrm{Ts}^{+}\right)$phenotype by Emc4p at $37^{\circ} \mathrm{C}$. 
Effect of Emc4 protein overexpression on $\mathrm{H}_{2} \mathrm{O}_{2}$-, ethanol-, and caffeine-mediated cell death of elF2B mutant and wild type $S$. cerevisiae strains

Three different sets of experiments (quantitative assay, spot assay, and halo assay) were performed. Wild-type GCD7 GCN2, GCD7 gcn2A, and mutant gcd7-201 gcn2 $\Delta$ strains of $S$. cerevisiae containing pEG(KG) or pEG(KG)/ Emc4 were incubated for $16 \mathrm{~h}$ at $30^{\circ} \mathrm{C}$ with shaking in the $\mathrm{SC}$-medium supplemented with either $2 \%$ galactose or raffinose (lacking uracil). SC-medium also contained $4 \mathrm{mM} \mathrm{H}_{2} \mathrm{O}_{2}$ [30], $10 \%$ ethanol [31], $20 \mathrm{mM}$ caffeine [32], 1.6\% DMSO [23], $35 \mathrm{mM}$ Dithiothreitol (DTT) [33], and $1 \mathrm{M} \mathrm{NaCl}$ [34] separately. After $16 \mathrm{~h}$ of growth, cell density was measured at $\mathrm{A}_{600} \mathrm{~nm}$ using a UV visible spectrophotometer. Spot and halo assays were performed as given by $[35,36]$.

Ten-fold serially diluted cultures were spotted to check Slg and Gcd phenotypes on SC-medium supplemented with either $2 \%$ galactose or raffinose (lacking uracil). The medium was also supplemented $30 \mathrm{mM} \mathrm{3-}$ AT for $\mathrm{Gcd}^{-}$phenotype, $4 \mathrm{mM} \mathrm{H}_{2} \mathrm{O}_{2}, 10 \%$ ethanol, 20 $\mathrm{mM}$ caffeine, 1.6\% DMSO, $35 \mathrm{mM}$ Dithiothreitol (DTT), and $1 \mathrm{M} \mathrm{NaCl}$. The plates were incubated at $30^{\circ} \mathrm{C}$ for 2 days and observed for pattern of yeast cell growth.

For halo assay, filter disks containing $4 \mathrm{mM} \mathrm{H}_{2} \mathrm{O}_{2}$, $10 \%$ ethanol, $20 \mathrm{mM}$ caffeine, $1.6 \%$ DMSO, $35 \mathrm{mM}$ Dithiothreitol (DTT), and $1 \mathrm{M} \mathrm{NaCl}$ were placed on SC agar medium supplemented with either $2 \%$ galactose or raffinose (lacking uracil) with uniformly spread culture of $S$. cerevisiae mutant and wild-type strains containing pEG(KG) or pEG(KG)/Emc4. Plates were incubated at $30^{\circ} \mathrm{C}$ for 2 days and observed for zone of inhibition. The S.cerevisiae strains GCD7 GCN2, GCD7 gcn $2 \Delta$, and gcd7-201 gcn2 2 (Supplementary Table 1) were streaked on YPD plates with or without $\mathrm{H}_{2} \mathrm{O}_{2}$, ethanol, caffeine, DMSO, Dithiothreitol (DTT), and $\mathrm{NaCl}$.

\section{Results}

Screening of genomic DNA library clones for rescuing slow growth phenotype of $S$. cerevisiae elF2B mutant strains

Approximately, 30 transformants ( $g c d 7-201 \operatorname{gcn} 2 \Delta$ transformants) were observed, showing colony size equivalent to that of isogenic wild-type GCD7 gcn $2 \triangle$ transformed with vector alone were screened further for $\mathrm{Gcd}^{+}$phenotype. Out of 30 transformants, only the transformant named as Sup-I restored the growth of $g c d 7-201 \operatorname{gcn} 2 \Delta$ as well as $\mathrm{Gcd}^{-}$phenotype. The growth of Sup-I clone was very similar to that of isogenic wild-type GCD7 $g c n 2 \Delta$ transformed with empty vector (Figure S1 a and b).

Plasmid DNA was rescued from Sup-I clone (Figure S1 c) and transformed into $g c d 7-201 g c n 2 \Delta$ strain (Figure S1 d). The growth phenotypes $\left(\mathrm{Slg}^{+}\right.$and $\left.\mathrm{Gcd}^{+}\right)$of transformants were checked further by streaking mutant transformants
( $g c d 7-201 \quad g c n 2 \Delta)$ along with isogenic wild-type strain (Figure S1 d). Results revealed that $g c d 7-201$ $g c n 2 \Delta$ transformants were of uniform size and showed $\mathrm{Slg}^{+}$and $\mathrm{Gcd}^{+}$phenotype (S1 d and e). This data clearly suggests that a genomic clone (Sup-I) suppressed the $\mathrm{Slg}^{-}$and $\mathrm{Gcd}^{-}$phenotype of $\operatorname{gcd} 7-201$ gcn $2 \Delta$ mutant strain.

The Sup-I clone was sequenced using YEp24 specific primers (Table S7). The Sup-I genomic construct revealed the presence of only two complete ORFs including EMC4 $573 \mathrm{bp}$ and YGL230C 444 bp genes encoding for chaperone and putative protein respectively, whereas TAN1 (YGL232W) and SAP4 genes were truncated. This data suggests that Sup-I harbors full length EMC4 that rescued slow growth and $\mathrm{Gcd}^{-}$ phenotype of $g c d 7-201 \operatorname{gcn} 2 \Delta$ (Fig. 1a).

\section{Sub-cloning of potential suppressor gene into yeast expression vector, $\mathrm{pEG}(\mathrm{KG})$}

$E M C 4$ gene was amplified using gene-specific primers (Table S3) containing XbaI restriction site in the forward primer and SalI in the reverse primer followed by cloning in $\mathrm{pEG}(\mathrm{KG})$ vector at respective restriction sites. As expected, PCR product of $\sim 0.55 \mathrm{~kb}$ was observed on agarose gel (Figure S2 b). EMC4 gene is present on chromosome VII of $S$. cerevisiae genome (http://www. yeastgenome.org/) (S2 a). pEG(KG) vector of $9.3 \mathrm{~kb}$ containing GST tag under GAL1 promoter was used for sub-cloning of EMC4 gene (Figure S2 c). EMC4 gene sequence was verified by sequencing, and error free and complete sequence of $573 \mathrm{bp}$ was obtained.

\section{GST-Emc4 expression rescued the $\mathrm{Slg}^{+}$and $\mathrm{Gcd}^{+}$ phenotype of $\operatorname{gcd} 7-201 \mathrm{gcn} 2 \Delta$}

The $g c d 7-201 \quad g c n 2 \Delta$ was transformed with vector $\mathrm{pEG}(\mathrm{KG})$ alone or with GST-EMC4 expression construct, and the transformants were streaked on SC medium without uracil but supplemented with either raffinose (Fig. 1a) or galactose (Fig. 1b). As expected, gcd7-201 gcn2 2 transformed with vector alone or EMC4 construct showed slow growth phenotype on SC medium containing raffinose. Interestingly, GST-Emc4 rescued the growth of $g c d 7-201 \operatorname{gcn} 2 \Delta$, when streaked on SC medium containing galactose (Fig. 1b).

To further analyze the results, GST-EMC4, a uracilbased plasmid, was evicted on FOA-containing medium and showed original slow growth phenotype $g c d 7-201$ gcn2 2 (Fig. 1c) but cannot grow on SC medium without uracil supplementation (Fig. 1d). This data clearly suggests that overexpression of Emc4 rescued the $\mathrm{Slg}^{+}$of gcd7-201 gcn2 $\Delta$ mutant.

Further, GST-Emc4 was analyzed for rescuing $\mathrm{Gcd}^{+}$ phenotype of $\operatorname{gcd} 7-201 \operatorname{gcn} 2 \Delta$ mutant strain (Fig. 1e). As expected, $g c d 7-201 g c n 2 \Delta$ transformed with vector alone 


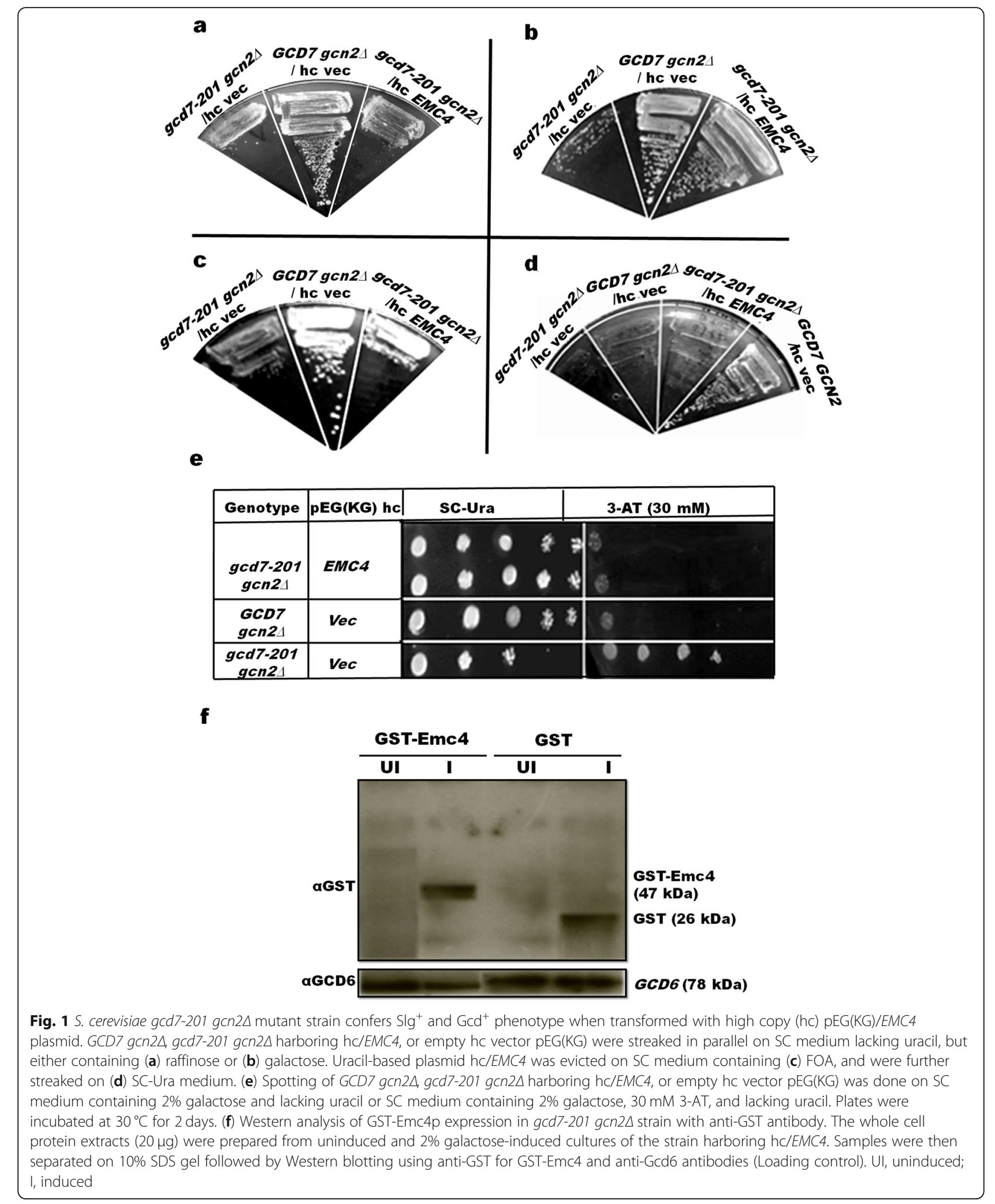

showed slow growth phenotype in SC medium without uracil supplementation as well as $\mathrm{Gcd}^{-}$phenotype on medium containing 3-AT. $\mathrm{Gcd}^{+}$phenotype of $\operatorname{gcd} 7-201$ $g c n 2 \Delta$ was also rescued when GST-Emc4 was expressed. The suppression of $\mathrm{Slg}^{-}$and $\mathrm{Gcd}^{-}$phenotype by overexpression of GST-Emc4 suggests that Emc4 is involved 


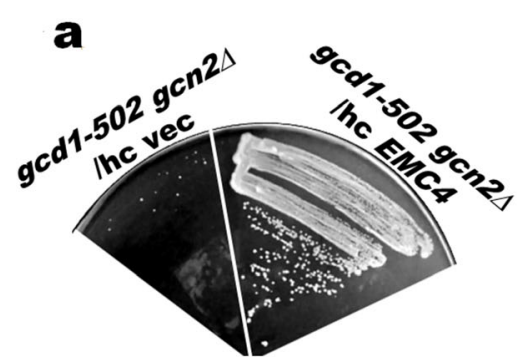

b

\begin{tabular}{|c|c|c|c|}
\hline Genotype & pEG(KG) hc & SC-Ura & 3-AT (30 mM) \\
\hline gcd1-502 gcn2 & Vec & & $\forall-16$ \& \\
\hline $\operatorname{gcd1-502} \operatorname{gcn} 2 \Delta$ & $E M C 4$ & & 9 \\
\hline
\end{tabular}

Fig. 2 Effect of Emc4p expression on elF2B mutants. gcd1-502 gcn2 $\Delta$ mutant strain rescued $\mathrm{Ts}^{+}, \mathrm{Slg}^{+}$, and $\mathrm{Gcd}^{+}$phenotype when transformed with hc/EMC4. (a) gcd1-502 gcn2 $\triangle$ transformants showing Slg ${ }^{+}$phenotype at $37^{\circ} \mathrm{C}$ were streaked along with gcd1-502 gcn2 $\Delta$ transformed with hc vector, pEG (KG). (b) Serial dilutions of the transformants showing Slg ${ }^{+}$phenotype were spotted on SC-Ura and SC medium containing $30 \mathrm{mM}-$ 3AT. gcd1-502 gcn2 $\triangle$ transformed with hc vector, pEG(KG), was spotted as a control. Plates were incubated for 2 days at $30^{\circ} \mathrm{C}$

directly or indirectly in eIF2B-mediated translation regulation.

The expression of GST-Emc4 was verified by western blotting. Whole cell extracts (WCE) of $g c d 7-201 \operatorname{gcn} 2 \Delta$ mutant expressing GST-Emc4 or GST alone were observed using anti-GST antibodies. Interestingly, a band of $26 \mathrm{kDa}$ of GST protein was detected in extracts expressing GST alone, while a band of $\sim 47 \mathrm{kDa}$ protein was detected in WCE of $g c d 7-201$ gcn $2 \Delta$ mutant transformed with GST-Emc4 construct. $\alpha$-GCD6 antibodies were used as internal loading control (Fig. 1f).

\section{Strain specific suppression of elF2B mutations by Emc4p} Originally, Emc4p was isolated as suppressor of $g c d 7$ 201 mutation of eIF2B $\beta$. The effect of overexpression of Emc4p on other eIF2B mutations including, $g c d 6-1$ gcn2A, gcd1-502 gcn2-101, gcd12-503 gcn2-101, and GCN2 mutant gcn2::LEU2 (Table S2) was also tested. Interestingly, the Emc4p overexpression rescued temperature sensitive $\left(\mathrm{Ts}^{-}\right)$phenotype of $\operatorname{gcd} 1-502$ at $37^{\circ} \mathrm{C}$ (Fig. 2a), but not that of other eIF2B mutants (data not shown). Emc4p overexpression also rescued $\mathrm{Slg}^{-}$and $\mathrm{Gcd}^{-}$phenotype of $\operatorname{gcd} 1-502$ gcn2-101 (Fig. 2b). These results suggested that Emc4p causes strain-specific suppression of eIF2B mutants.

\section{GST-Emc4 expression enhances stress tolerance in S. cerevisiae} GST-Emc4 overexpression protects wild type and mutant S. cerevisiae strains ( gcd7-201 gcn2 2 , GCD7 gcn $2 \Delta$, and GCD7 GCN2) against $4 \mathrm{mM} \mathrm{H}_{2} \mathrm{O}_{2}, 10 \%$ ethanol, and $30 \mathrm{mM}$ caffeine-mediated stress. $g c d 7-201 \operatorname{gcn} 2 \Delta$, GCD7 gcn2 2 , and GCD7 GCN2 streaked on YPD agar plates showed $\mathrm{Slg}^{-}$phenotype under $\mathrm{H}_{2} \mathrm{O}_{2}$, ethanol, and caffeine stress (Figure S3 a-c). The $S$. cerevisiae gcd7-201 $g c n 2 \triangle, G C D 7 g c n 2 \Delta$, and GCD7 GCN2 transformed with vector alone showed reduced growth (2-fold) under 4 $\mathrm{mM} \mathrm{H}_{2} \mathrm{O}_{2}, 10 \%$ ethanol, and $30 \mathrm{mM}$ caffeine stress in presence of either raffinose or galactose. In contrast, $\operatorname{gcd} 7-201 \operatorname{gcn} 2 \Delta$, GCD7 $g c n 2 \Delta$, and GCD7 GCN2 transformed with pEG(KG)/EMC4 showed normal growth phenotype even under $\mathrm{H}_{2} \mathrm{O}_{2}$, ethanol, and caffeine stress in presence of $2 \%$ galactose (Figure S3 a-c ). Emc4p overexpression protects $S$. cerevisiae to $\mathrm{H}_{2} \mathrm{O}_{2}$, ethanol-, and caffeine-induced cell death. In contrast, no significant protective effect was observed against DMSO, $\mathrm{NaCl}$, and DTT (data not shown).

In spot assays, the $S$. cerevisiae cells $\operatorname{gcd} 7-201 \operatorname{gcn} 2 \Delta$, GCD7 gcn $2 \triangle$, and GCD7 GCN2 overexpressing GSTEmc4 rescued the growth following exposure to $\mathrm{H}_{2} \mathrm{O}_{2}$, caffeine, and ethanol in presence of $2 \%$ galactose compared to control cells (Fig. 3a-c).

The halo assay reveals that the zone of no growth surrounding the hydrogen peroxide, caffeine, and alcohol containing filter was significantly reduced in $g c d 7-201$ gcn $2 \triangle$, GCD7 gcn $2 \Delta$, and GCD7 GCN2 strains overexpressing GST-Emc4 in presence of $2 \%$ galactose as compared to control cells (Fig. 4a-c). This study strongly suggests that overexpression of Emc4p is capable of preventing cell death caused by high concentrations of $\mathrm{H}_{2} \mathrm{O}_{2}$, alcohol, and caffeine. Moreover, overexpression of Emc4p repairs $g c d 7-201$ gcn $2 \Delta$-based defect in translation initiation.

Based upon the results, we proposed a schematic model (Figure S4) describing the effect of overexpression of Emc4p on eIF2B mutations. During amino acid starvation, $\mathrm{H}_{2} \mathrm{O}_{2}$, ethanol, and caffeine stress in S. cerevisiae (wild type), Gcn4p is derepressed. eIF2B mutations are known to derepress the Gcn $4 p$ independent of Gcn2p [53]. According to the present model, Emc4p (chaperone protein) overexpression might stabilize the unstable eIF2B complex by properly folding the misfolded subunits of eIF2B complex due to its chaperone activity, which results into stable eIF2-eIF2B interaction and initiates eIF2B GEF activity. This model also describes the role of Emc4p in stress response, where Emc4p overexpression mediates expression of stress response genes under amino acid starvation, $\mathrm{H}_{2} \mathrm{O}_{2}$, ethanol, and caffeine stress. 


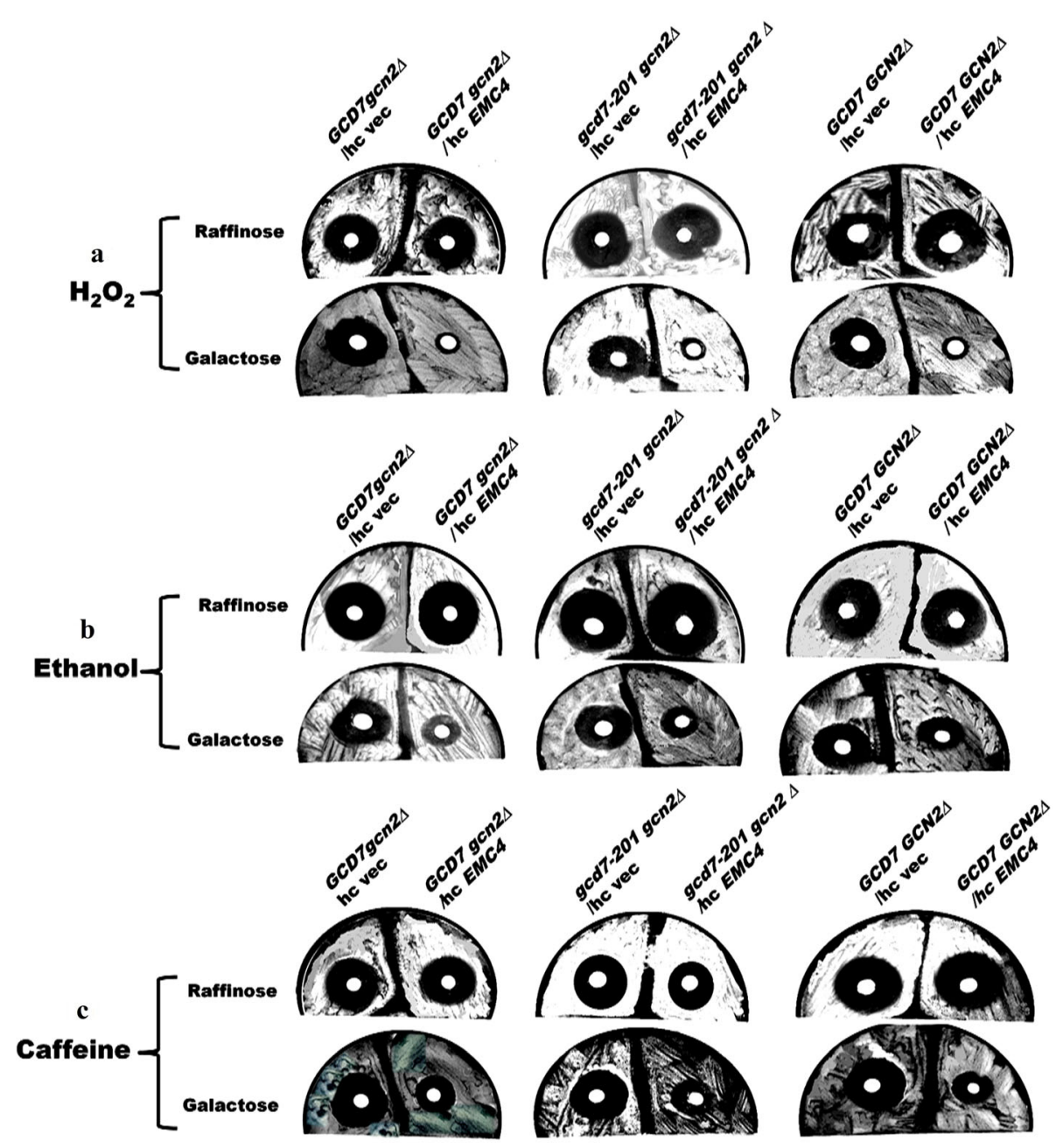

Fig. 3 Halo assay to test the effect of $\mathrm{H}_{2} \mathrm{O}_{2}$, ethanol, and caffeine on the growth of elF2B mutants. Hydrogen peroxide, ethanol, and caffeine halo assays were performed with gcd7-201 gcn2 $\triangle$ GCD7 gcn2 4 and GCD7 GCN2. S. cerevisiae strains harboring either the empty hc vector, pEG (KG), or the hc/EMC4 gene expressing plasmid were spread on SC medium, containing filter disks soaked in (a) $\mathrm{H}_{2} \mathrm{O}_{2}(4 \mathrm{mM})$, (b) ethanol (10\%), and (c) caffeine $(30 \mathrm{mM})$ in the presence of either raffinose or galactose. The plates were incubated at $30^{\circ} \mathrm{C}$ for $2-3$ days

\section{Discussion}

Eukaryotic initiation factor $2 \mathrm{~B}$ (eIF2B) is involved in translation initiation/regulation in eukaryotes. Mutations in eIF2B genes lead to deregulation of translation initiation and regulation, causing vanishing white matter disease (VWM). The goal of this study was to identify the extragenic suppressors of $S$. cerevisiae eIF2B mutations corresponding to human eIF2B mutations and to study the role of suppressor protein in eIF2B-mediated regulatory pathways under different stress conditions. $S$. cerevisiae genomic DNA library was constructed and transformed into the eIF2B mutant strains for identification of extragenic suppressors of eIF2B mutations.

Emc4p was observed to suppress the growth defect of S. cerevisiae, caused by $g c d 7-201 \operatorname{gcn} 2 \Delta$ and $g c d 1-502$ gcn2-101 mutations. $g c d 7-201$ is a missense eIF2B $\beta^{\text {V341D }}$ mutation corresponding to human eIF2B $\beta^{\mathrm{V} 316 \mathrm{D}}$ causing improper folding of eIF2B $\beta$ subunit, as a result eIF2B $\delta$ is excluded from unstable eIF2B $\beta^{\mathrm{V} 341 \mathrm{D}}$ complexes, and eventually, rate of protein synthesis is also reduced [18]. Similar results have been reported in previous studies showing that $\beta$ subunit Gcd7p provides a platform for interaction with Gcd2p helping proper eIF2B complex formation [14]. $g c d 1-502$ is also a missense mutation of eIF2 $\gamma$ (L480Q). This mutation affects the $\gamma$ subunit of eIF2B and lowers its GDP dissociation factor (GDF) activity and translation [13].

Endoplasmic reticulum membrane complex subunit 4 (EMC4) is a member of endoplasmic reticulum transmembrane complex (EMC complex) and is characterized as a chaperone protein (null mutants are known to induce the unfolded protein response) [5,6]. This protein also plays an important role in biosynthesis of ionotropic acetylcholine receptors and its inactivation reduces the 


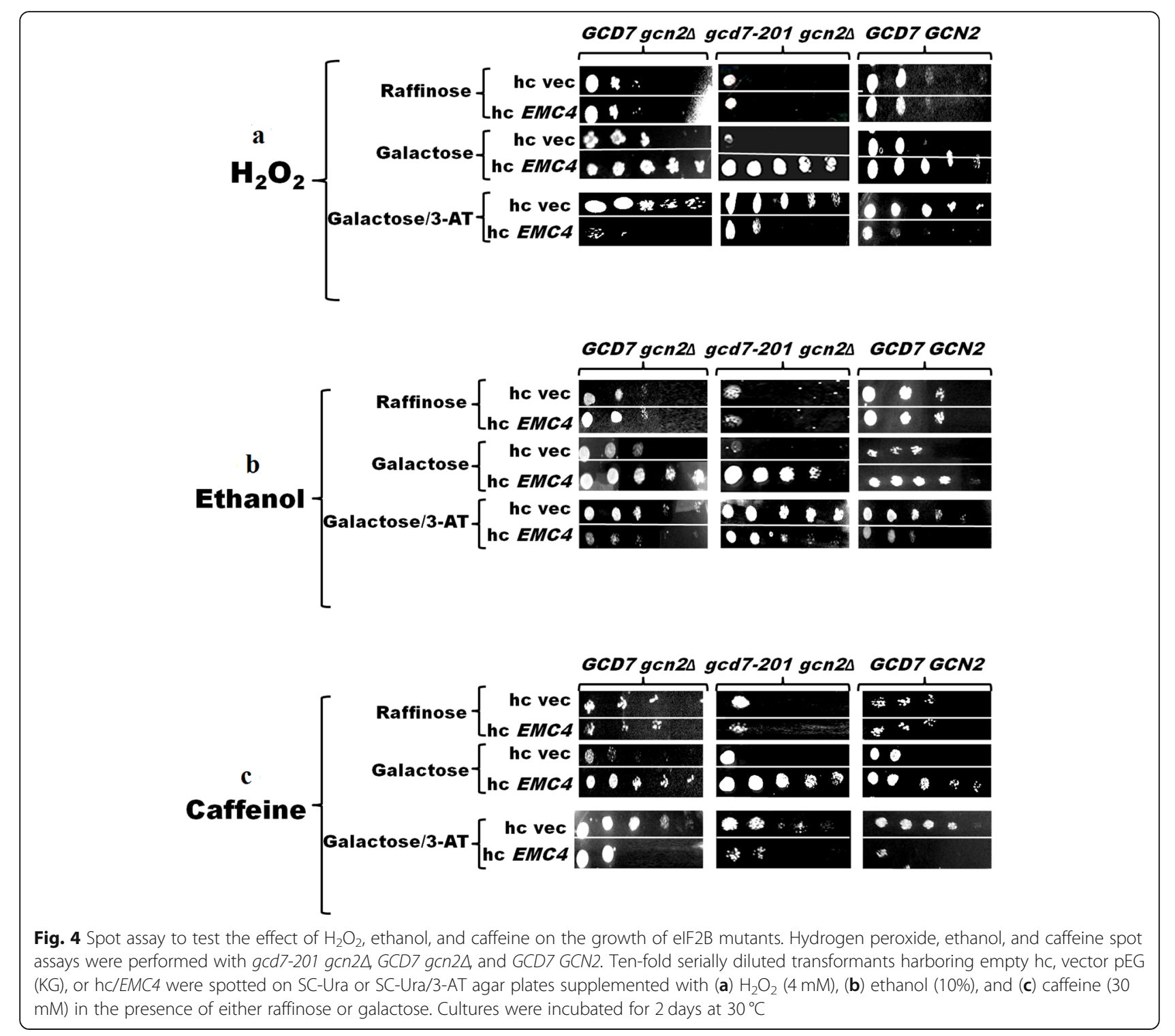

total number of nicotinic acetylcholine receptors (AChRs) present on plasma membrane of muscle and neuronal cells [33]. Thus, Emc4p is important for the development of muscle and neuronal cells. Slight change in the levels of Emc4p can cause brain-related disorders like vanishing white matter disease. But no relevance of Emc4p and VWM has been reported till now. Studies in mammalian systems have reported the high levels of EMC4 protein in the brain and specially during developmental stages of the organism (http://www.proteinatlas. org). Emc4p is also involved in stress response as described in a study which shows that overexpression of YGL230C inhibits the hydrogen peroxide-mediated oxidative stress and cell death in S. cerevisiae [30]. EMC4 is also involved in tethering of endoplasmic reticulum with mitochondria, important step for phospholipid metabolism. Lipids being important components of neuronal cells require more lipids, and defect in lipid metabolism in nerve cells can cause neurodegenerative disease [37]. These studies support our data that Emc4p is an important protein, regulating translation in some or other way in brain cells.

Emc4p is also involved in hydrogen peroxide-mediated oxidative stress response in S. cerevisiae [30]. Considering this fact, $g c d 7-201 \operatorname{gcn} 2 \Delta$, GCD7 $g c n 2 \Delta$, and GCD7 GCN2 strains of $S$. cerevisiae overexpressing Emc4p were also tested for resistance to other translation-inhibiting compounds $\left(\mathrm{H}_{2} \mathrm{O}_{2}\right.$, ethanol, caffeine, DMSO, DTT, and $\mathrm{NaCl}$ ). Surprisingly, Emc4p overexpression rescued both the $\mathrm{Slg}^{-}$and $\mathrm{Gcd}^{-}$phenotype of GCD7 GCN2, GCD7 gcn $2 \Delta$, and $g c d 7-201 \operatorname{gcn} 2 \Delta S$. cerevisiae strains under $\mathrm{H}_{2} \mathrm{O}_{2}$, ethanol, and caffeine stress but no effect was 
observed for DMSO-, DTT-, and NaCl-mediated stress conditions. Here we demonstrated that the overexpression of Emc4p suppressed the $\mathrm{H}_{2} \mathrm{O}_{2^{-}}$, ethanol-, and caffeine-mediated growth inhibition of $g c d 7-201 \operatorname{gcn} 2 \Delta$ mutant and wild-type strains in Gcn2p independent manner possibly by modulating/targeting the eIF2B for translational regulation. According to the present study, $\mathrm{H}_{2} \mathrm{O}_{2}$, ethanol, and caffeine might be used as chemical modulators to study eIF2B-mediated pathways leading to stress responses as described previously [16]. Earlier study has proposed that Tan1p overexpression confers resistance to GCD7 GCN2, $\operatorname{gcd} 7-201 \operatorname{gcn} 2 \Delta$, and GCD7 gcn $2 \Delta$ growth defect under ethanol, $\mathrm{H}_{2} \mathrm{O}_{2}$, and caffeine stress [38].

Our studies are consistent with the previous reports of Gcn2 independent Gcn4 induction and translation regulation. For illustration, role of butanol mediated induction of GCN4 by a Gcn2p-independent manner has already been reported [39]. Oxidative stress $\left(\mathrm{H}_{2} \mathrm{O}_{2}\right)$ causes translation inhibition by Gcn 2 or eIF $2 \alpha$ phosphorylation independent manner [40]. The molecular mechanisms of these processes are not fully understood.

Although, the mechanism by which Emc4p functions in translation regulation is still unknown, this study provides strong evidence that Emc4p protein in some or other way (possibly by stabilizing the eIF2eIF2B interaction) is involved in the eIF2B-mediated translation initiation and regulation pathway. More importantly, the role of Emc4p in the brain describes and supports our study and could provide a tool for understanding the mechanism behind vanishing white matter disease.

\section{Conclusions}

In this work, we identified Emc4p as an extragenic suppressor of eIF2B mutations $(g c d 7-201 \operatorname{gcn} 2 \Delta$ and gcd-502 gcn $2 \Delta$ ). Our results revealed that Emc4p suppresses the slow growth and general control derepression phenotypes of $S$. cerevisiae eIF2B mutations, corresponding to human eIF2B mutations. Emc4p does this by its chaperone activity in which it properly folds and stabilizes the mutant subunits $(g c d 7$ 201 and $g c d 1-502)$. This indicates that interaction of these two subunits is important for eIF2B activity. In addition, Emc4p also suppresses the $S$. cerevisiae growth defect under $\mathrm{H}_{2} \mathrm{O}_{2}$, ethanol, and caffeine stress which clearly indicates the role of Emc4p in eIF2B-mediated translation initiation and regulation most importantly in the brain. Our results help in understanding the mechanism behind VWM disease as our results are supported by previous studies in which the different roles of Emc4p in the brain and in stress response are clearly described.

\section{Supplementary information}

Supplementary information accompanies this paper at https://doi.org/10. 1186/s43141-020-00029-7.

Additional file 1. Supplementary file

\section{Abbreviations}

WWM: vanishing white matter; elF2B: Eukaryotic initiation factor 2B; EMC4: ER membrane protein complex subunit 4; Slg: slow growth; Gcd: general control derepression; 3-AT: 3-Amino triazole; SC: synthetic complete medium; Cfu: Colony forming units

\section{Acknowledgements}

Authors are thankful to Dr. Alan G. Hinnebusch, NICHD, USA, for providing yeast strains, a-GCD6 antibodies, and plasmids used in this study. Authors are also thankful to Shoolini University, Bajhol, Solan, Himachal Pradesh, India, for providing financial and infrastructural support for this study and all the members of Yeast Biology Lab for moral support.

\section{Authors' contributions}

SS performed all the experiments; KD and AS conceived the study and participated in the design of experiments. SS, AS, and KD wrote the manuscript, and DJB edited the manuscript. All authors read and approved the final manuscript.

\section{Funding}

No funding was received for this study. Shoolini University, Solan, HP, India, provided infrastructural and consumables support for the study.

Availability of data and materials

Data provided as supplementary.

Ethics approval and consent to participate

Not applicable.

Consent for publication

Not applicable.

\section{Competing interests}

The authors declare that they have no competing interests.

\section{Author details}

${ }^{1}$ Faculty of Applied Sciences and Biotechnology, Shoolini University, Solan, Himachal Pradesh 173212, India. ${ }^{2}$ Department of Food Science and Nutrition, University of Minnesota-Twin Cities, St. Paul, MN, USA. ${ }^{3}$ Microbial and Plant Genomic Institute, University of Minnesota-Twin Cities, St. Paul, MN, USA. ${ }^{4}$ Biotechnology Institute, University of Minnesota-Twin Cities, St. Paul, MN, USA.

Received: 25 February 2020 Accepted: 22 April 2020

Published online: 01 June 2020

\section{References}

1. Wortham NC, Proud CG (2015) Biochemical effects of mutations in the gene encoding the alpha subunit of eukaryotic initiation factor (elF) 2B associated with vanishing white matter disease. BMC Med Genet 16:64 https://doi.org/ 10.1186/s12881-015-0204-z

2. Pavitt GD, Ramaiah KV, Kimball SR, Hinnebusch AG (1998) elF2 independently binds two distinct elF2B subcomplexes that catalyze and regulate guanine-nucleotide exchange. Genes Dev 12:514-526 https://doi. org/10.1101/gad.12.4.514

3. Krishnamoorthy T, Pavitt GD, Zhang F, Dever TE, Hinnebusch AG (2001) Tight binding of the phosphorylated a subunit of initiation factor 2 (elF2a) to the regulatory subunits of guanine nucleotide exchange factor elF2B is required for inhibition of translation initiation. Mol Cell Biol 21:5018-5030 https://doi.org/10.1128/mcb.21.15.5018-5030.2001

4. Rowlands AG, Panniers R, Henshaw EC (1988) The catalytic mechanism of guanine nucleotide exchange factor action and competitive inhibition by phosphorylated eukaryotic initiation factor 2. J Biol Chem 263:5526-5533 
5. Harding HP, Novoa I, Zhang Y, Zeng H, Wek R, Schapira M, Ron D (2000) Regulated translation initiation controls stress-induced gene expression in mammalian cells. Mol Cell 6: 1099-1108. https://doi.org/10.1016/S10972765(00)00108-8.

6. Hinnebusch AG (1997) Translational regulation of yeast GCN4. A window on factors that control initiator-tRNA binding to the ribosome. J Biol Chem 272 . 21661-21664 https://doi.org/10.1074/jbc.272.35.21661

7. Natarajan K, Meyer MR, Jackson BM, Slade D, Roberts C, Hinnebusch AG, Marton MJ (2001) Transcriptional profiling shows that Gcn4p is a master regulator of gene expression during amino acid starvation in yeast. Mol Cell Biol 21:4347-4368 https://doi.org/10.1128/mcb.21.13.4347-4368.2001

8. Fogli A, Rodriguez D, Eymard-Pierre E, Bouhour F, Labauge P, Meaney BF, Zeesman S, Kaneski CR, Schiffmann R, Boespflug-Tanguy O (2003) Ovarian failure related to eukaryotic initiation factor $2 B$ mutations. Am J Hum Genet 72:1544-1550 https://doi.org/10.1086/375404

9. Schiffmann R, Elroy-Stein O (2006) Childhood ataxia with CNS hypomyelination/vanishing white matter disease-a common leukodystrophy caused by abnormal control of protein synthesis. Mol Genet Metab 88:7-15 https://doi.org/10.1016/j.ymgme.2005.10.019

10. Pronk JC, Van Kollenburg B, Scheper GC, Van Der Knaap MS (2006) Vanishing white matter disease: a review with focus on its genetics. Ment Retard Dev Disabil Res Rev 12(2):123-128 https://doi.org/10.1002/mrdd. 20104

11. Horzinski L, Huyghe A, Cardoso MC, Gonthier C, Ouchchane L, Schiffmann R, Blanc P, Boespflug-Tanguy O, Fogli A (2009) Eukaryotic initiation factor 2B (elF2B) GEF activity as a diagnostic tool for EIF2B-related disorders. PLoS One 4(12):e8318 https://doi.org/10.1371/journal.pone.0008318

12. Hannig EM, Williams NP, Wek RC, Hinnebusch AG (1990) The translational activator GCN3 functions downstream from GCN1 and GCN2 in the regulatory pathway that couples GCN4 expression to amino acid availability in Saccharomyces cerevisiae. Genetics 126:549-562

13. Dever TE, Chen JJ, Barber GN, Cigan AM, Feng L, Donahue TF, London IM, Katze MG, Hinnebusch AG (1993) Mammalian eukaryotic initiation factor 2 alpha kinases functionally substitute for GCN2 protein kinase in the GCN4 translational control mechanism of yeast. Proc Natl Acad Sci 90:4616-4620 https://doi.org/10.1073/pnas.90.10.4616

14. Vazquez de Aldana CR, Hinnebusch AG (1994) Mutations in the GCD7 subunit of yeast guanine nucleotide exchange factor elF-2B overcome the inhibitory effects of phosphorylated elF-2 on translation initiation. Mol Cell Biol 14:3208-3222 https://doi.org/10.1128/mcb.14.5.3208

15. Pavitt GD, Yang W, Hinnebusch AG (1997) Homologous segments in three subunits of the guanine nucleotide exchange factor elF2B mediate translational regulation by phosphorylation of elF2. Mol Cell Biol 17:12981313 https://doi.org/10.1128/mcb.17.3.1298

16. Fogli A, Boespflug-Tanguy $O$ (2006) The large spectrum of elF2B-related diseases. Biochem Soc Trans 34(1):22-29 https://doi.org/10.1042/BST0340022

17. Leegwater PA, Vermeulen G, Könst AA, Naid S, Mulders J, Visser A, Kersbergen P, Mobach D, Fonds D, van Berkel CG, Lemmers RJ (2001) Subunits of the translation initiation factor elF2B are mutant in leukoencephalopathy with vanishing white matter. Nat Genet 29:383-388 https://doi.org/10.1038/ng764

18. Richardson JP, Mohammad SS, Pavitt GD (2004) Mutations causing childhood ataxia with central nervous system hypomyelination reduce eukaryotic initiation factor $2 \mathrm{~B}$ complex formation and activity. Mol Cell Biol 24:2352-2363 https://doi.org/10.1128/mcb.24.6.2352-2363.2004

19. van der Knaap MS, Leegwater PA, Könst AA, Visser A, Naidu S, Oudejan C, Schutgens RB, Pronk JC (2002) Mutations in each of the five subunits of translation initiation factor elF2B can cause leukoencephalopathy with vanishing white matter. Ann Neurol 51:264-270 https://doi.org/10.1002/ana. 10112

20. Dev K, Santangelo TJ, Rothenburg S, Neculai D, Dey M, Sicheri F, Dever TE, Reeve JN, Hinnebusch AG (2009) Archaeal alF2B interacts with eukaryotic translation initiation factors elF2 $a$ and elF2Ba: implications for alF2B function and elF2B regulation. J Mol Biol 392:701-722 https://doi.org/10. 1016/j.jmb.2009.07.030

21. Dev K, Qiu H, Dong J, Zhang F, Barthlme D, Hinnebusch AG (2010) The beta/Gcd7 subunit of eukaryotic translation initiation factor 2B (elF2B), a guanine nucleotide exchange factor, is crucial for binding elF2 in vivo. Mol Cell Biol 30:5218-5233 https://doi.org/10.1128/MCB.00265-10

22. Moon SL, Parker R (2018) EIF2B2 mutations in vanishing white matter disease hypersuppress translation and delay recovery during the integrated stress response. RNA. 24(6):841-852 (2018). https://doi.org/10.1261/rna. 066563.118.

23. Motlekar N, de Almeida RA, Pavitt GD, Diamond SL, Napper AD (2009) Discovery of chemical modulators of a conserved translational control pathway by parallel screening in yeast. Assay and drug development technologies 7:479-494 https://doi.org/10.1089/adt.2009.0198

24. Sambrook J, Fritsch E.F, Maniatis T (1989) Molecular cloning: a laboratory manual, 2nd ed., Vols. 1, 2 and 3.

25. Elble R (1992) A simple and eficient procedure for transformation of yeasts. BioTechniques 13(1):18-20

26. Rose MD, Novick P, Thomas JH, Botstein D, Fink GR (1987) A Saccharomyces cerevisiae genomic plasmid bank based on a centromere-containing shuttle vector. Gene 60 (2-3):237-243. https://doi.org/10.1016/0378-1119(87)90232-0.

27. Gunde T, Barberis A (2005) Yeast growth selection system for detecting activity and inhibition of dimerization-dependent receptor tyrosine kinase. BioTechniques. 39(4):541-549. https://doi.org/10.2144/000112011

28. Hoffman CS, Winston F (1987) A ten-minute DNA preparation from yeast efficiently releases autonomous plasmids for transformaion of Escherichia coli. Gene 57 (2-3): 67-272 . https://doi.org/10.1016/0378-1119(87)90131-4.

29. DJ DJSG, Zhang B, Kraemer B, Pochart P, Fields S, Wickens M (1996) A threehybrid system to detect RNA-protein interactions in vivo. Proc Natl Acad Sci U S A 93(16):8496-8501 https://doi.org/10.1073/pnas.93.16.8496

30. Ring G, Khoury CM, Solar AJ, Yang Z, Mandato CA, Greenwood MT (2008) Transmembrane protein 85 from both human (TMEM85) and yeast (YGL231c) inhibit hydrogen peroxide mediated cell death in yeast. FEBS Lett 582:2637-2642 https://doi.org/10.1016/j.febslet.2008.06.042

31. Yamauchi Y, Izawa S (2016) Prioritized expression of BTN2 of Saccharomyces cerevisiae under pronounced translation repression induced by severe ethanol stress. Front Microbiol 7:1319 https://doi.org/10.3389/fmicb.2016. 01319

32. Wanke V, Cameroni E, Uotila A, Piccolis M, Urban J, Loewith R, De Virgilio C (2008) Caffeine extends yeast lifespan by targeting TORC1. Mol Microbiol 69(1):277-285 https://doi.org/10.1111/j.1365-2958.2008.06292.x

33. Richard M, Boulin T, Robert VJ, Richmond JE, Bessereau JL (2013) Biosynthesis of ionotropic acetylcholine receptors requires the evolutionarily conserved ER membrane complex. Proc Natl Acad Sci 110(11):E1055-E1063 https://doi.org/10.1073/pnas.1216154110

34. Melamed D, Pnueli L, Arava Y (2008) Yeast translational response to high salinity: global analysis reveals regulation at multiple levels. RNA 14:13371351 https://doi.org/10.1261/rna.864908

35. Flower TR, Chesnokova LS, Froelich CA, Dixon C, Witt SN (2005) Heat shock prevents alpha-synuclein-induced apoptosis in a yeast model of Parkinson's disease. J Mol Biol 351:1081-1100 https://doi.org/10.1016/j.jmb.2005.06.060

36. Yang Z, Khoury C, Jean-Baptiste G, Greenwood MT (2006) Identification of mouse sphingomyelin synthase 1 as a suppressor of Bax-mediated cell death in yeast. FEMS Yeast Res 6:751-762 https://doi.org/10.1111/j.15671364.2006.00052.x

37. Lahiri S, Chao JT, Tavassoli S, Wong AKO, Choudhary V, Young BP, Loewen CJR, Prinz WA (2014) A conserved endoplasmic reticulum membrane protein complex (EMC) facilitates phospholipid transfer from the ER to mitochondria. PLoS Biol 12(10):e1001969 https://doi.org/10.1371/journal. pbio.1001969

38. Sharma S, Sourirajan A, Dev K (2017) Role of Saccharomyces cerevisiae TAN1 (tRNA acetyltransferase) in eukaryotic initiation factor 2B (elF2B) mediated translation control and stress response. 3 Biotech.7(3), 223.

39. Ashe MP, Slaven JW, Susan K, Ibrahimo S, Sachs AB (2001) A novel elF2Bdependent mechanism of translational control in yeast as a response to fusel alcohols. EMBO J 20:6464-6474 https://doi.org/10.1093/emboj/20.22. 6464

40. Knutsen JHJ, Rødland GE, Bøe CA, Håland TW, Sunnerhagen P, Grallert B, Boye $E$ (2015) Stress-induced inhibition of translation independently of elF2a phosphorylation. J Cell Sci 128:4420-4427 https://doi.org/10.1242/jcs. 176545

\section{Publisher's Note}

Springer Nature remains neutral with regard to jurisdictional claims in published maps and institutional affiliations. 